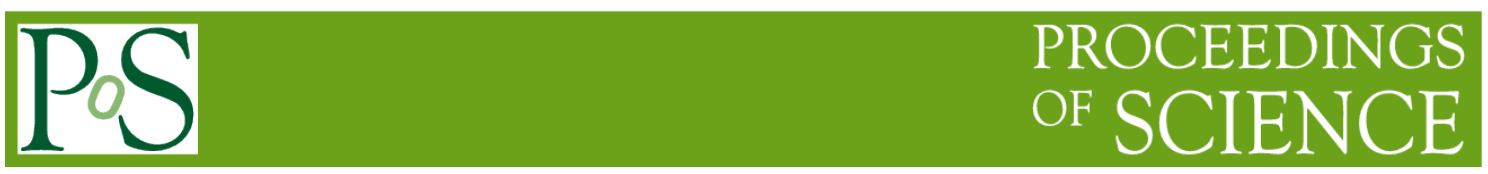

\title{
Associated production of Quarkonia and Heavy Hadrons
}

\author{
Jane Nachtman ${ }^{1}$ \\ University of Iowa \\ Iowa City, Iowa, USA \\ E-mail: jane-nachtman@uiowa.edu
}

Quarkonium production has long been considered an ideal means of investigating QCD and other new phenomena. Measurements of simultaneous production of quarkonium and other states are important for the understanding of the production mechanism; e.g. SPS (single parton scattering) versus DPS (double parton scattering). Measurements from ATLAS, CMS, LHCb, and D0 are reported.

38th International Conference on High Energy Physics

3-10 August 2016

Chicago, USA

\section{Introduction}

Measurements of quarkonium production, including double quarkonium or simultaneous production with W/Z provides insight into particle production at LHC/Tevatron. Experimental observation is needed to discern production through Single Parton Scattering (SPS) or Double Parton Scattering (DPS). Angular correlations between particles can be used to differentiate

\footnotetext{
${ }^{1}$ Speaker, supported by the U.S. Department of Energy Office of Science award DESC0010113.
} 
between models, but high-statistics datasets are needed. SPS models predict strongly correlated (small $|\Delta y|$ ) and DPS models predict less correlated (large $|\Delta y|)$ particle distributions. Events with $J / \psi, Y, W / Z$ provide clean signatures for probing the differences between production at the Tevatron and the LHC. Another motivation is the search for resonances or other exotic states such as tetraquarks, using quarkonium plus $\mathrm{X}$.

\section{$1.1 \mathrm{~J} / \Psi$ and $\mathrm{Y}$ production at colliders}

At colliders such as the LHC and the Tevatron, $J / \psi$, Y are "standard candles" for charm and b-physics studies, with their copious production described by Non-Relativistic QCD and providing input to refinement of the models. Decays to muon-anti-muon pairs are most commonly used to tag $\mathrm{J} / \psi, \psi(2 \mathrm{~S}), \mathrm{Y}, \mathrm{Y}(2 \mathrm{~S})$, and studies have been done at the LHC at 7, 8 and now $13 \mathrm{TeV}$. A key component of analyses featuring $\mathrm{J} / \psi$ is the ability to discriminate between prompt and non-prompt production using the pseudo-proper decay time, based on the transverse decay length and measurements of the dimuon invariant mass and $\mathrm{p}_{\mathrm{T}}$ Measurements were reported in [1],[2],[3].

\subsection{Double $J / \psi$ and $Y$ production}

Measurements of of $\mathrm{J} / \psi \mathrm{J} / \psi, \mathrm{J} / \psi \mathrm{Y}$ and YY can provide input on DPS and SPS contributions; production of $\mathrm{J} / \psi \mathrm{J} / \psi$ has been measured by (CMS, LHCb, D0)[4][5][6]. Analyses are guided by maximizing acceptance and minimizing model dependence by using $\mathrm{p}_{\mathrm{T}}$ thresholds to define the acceptance region. The CMS measurement of the differential cross section for prompt $\mathrm{J} / \psi \mathrm{J} / \psi$ is shown in Figure 1; the plot on the right shows the rapidity difference between the $\mathrm{J} / \psi$ 's; the large value at high $\Delta \mathrm{y}$ supports DPS models. The right plot shows the CMS analysis' ability to probe the high $\mathrm{p}_{\mathrm{T}}$ region.
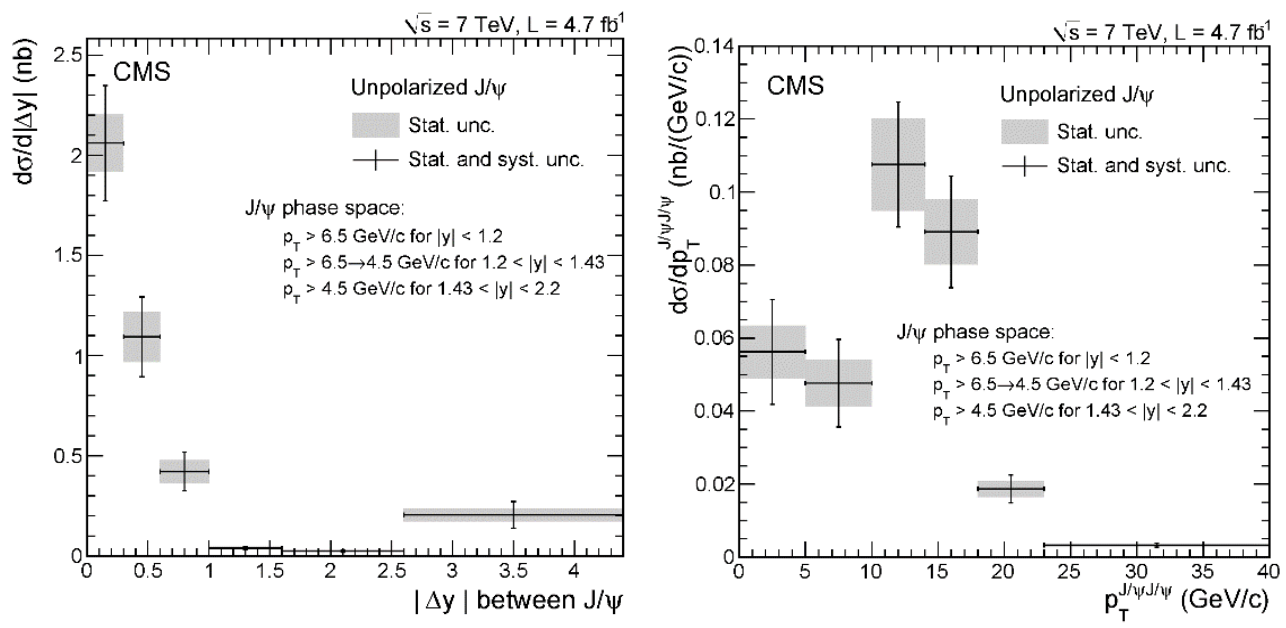

Figure 1: Differential cross section measurements from CMS, showing the prompt $J / \psi$ $\mathrm{J} / \psi$ production as a function of the rapidity difference (left) and the $\mathrm{p}_{\mathrm{T}}$ of the $\mathrm{J} / \psi \mathrm{J} / \psi$ system. 
The techniques developed for the $\mathrm{J} / \psi \mathrm{J} / \psi$ analysis can be transferred to isolate $\mathrm{J} / \psi \mathrm{Y}$ production, as shown in an analysis from the D0 collaboration. The D0 analysis [7] has enough statistics to analyze the angular correlations, as shown along with Monte Carlo models for SPS and DPS production, in Figure 2. This analysis shows that DPS is the favored interpretation.

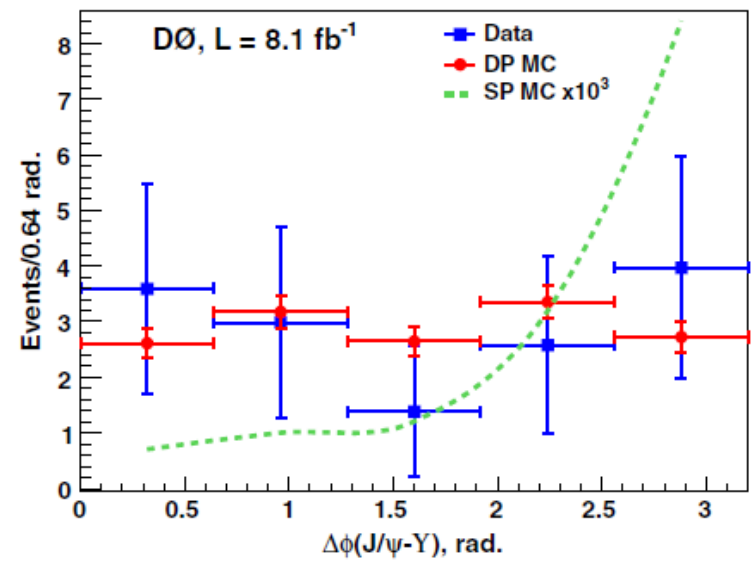

Figure 2: Angular correlations in the D0 J/ $\psi$ Y analysis. Plot from Reference [7].

CMS has made a first observation of simultaneous YY production[8] in the dimuon decay mode with a local significance greater than $5 \sigma$. No polarization is assumed; however, it is taken into account as a systematic uncertainty. The CMS analysis observes $38+/-7$ events and measures a cross section of

$$
\sigma(p p \rightarrow Y Y)=68.8 \pm 12.7(\text { stat }) \pm 7.4(\text { syst }) \pm 2.8(B R) p b \quad \text { as shown in Figure } 3 \text {. }
$$
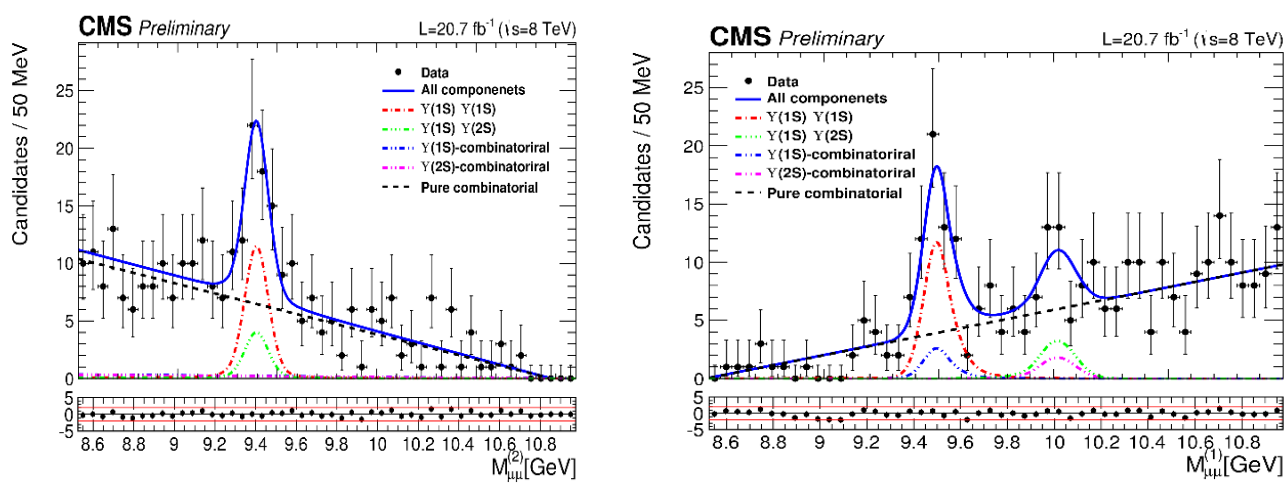

Figure 3. Dimuon mass plots from the CMS YY observation [8]. The figure on the left shows the lower dimuon mass and the figure on the right shows the higher dimuon mass from the final data sample.

\subsection{Y plus open charm production}

LHCb has published a suite of analyses examining Y plus open charm [9], where they measure combinations of $\mathrm{Y}(1 \mathrm{~S}), \mathrm{Y}(2 \mathrm{~S}), \mathrm{Y}(3 \mathrm{~S})$ and $\mathrm{D}^{0}, \mathrm{D}^{+}, \mathrm{D}^{+}{ }_{\mathrm{s}} \Lambda_{\mathrm{c}}$ They observe $\mathrm{Y}(1 \mathrm{~S}) \mathrm{D}^{0}$, 
$\mathrm{Y}(2 \mathrm{~S}) \mathrm{D}^{0}, \mathrm{Y}(1 \mathrm{~S}) \mathrm{D}^{+}, \mathrm{Y}(2 \mathrm{~S}) \mathrm{D}^{+}$and $\mathrm{Y}(1 \mathrm{~S}) \mathrm{D}_{\mathrm{s}}^{+}$at $>5 \sigma$ significance in 7 and $8 \mathrm{TeV}$ data. Measured cross sections and kinematics agree with a DPS model prediction.

\section{$1.4 \mathrm{~J} / \Psi$ plus $\mathrm{W} / \mathrm{Z}$}

ATLAS has analyzed production of $\mathrm{J} / \Psi$ plus $\mathrm{W} / \mathrm{Z}$ [10]. These processes provide a probe of DPS versus SPS and also give insight into color singlet or octet models.

Furthermore, these signatures can arise as potential rare higgs decays or other new physics signatures. The angular distributions are shown in Figure 4; the data agrees with the SPS model of production and does not agree well with DPS models.
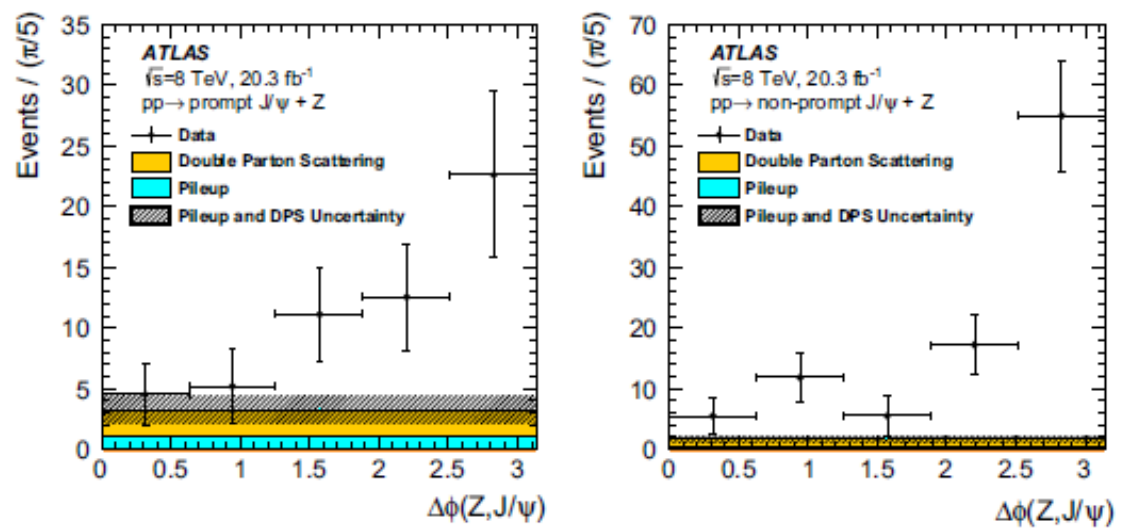

Figure 4. ATLAS data showing angular correlations in $J / \psi$ plus $W / Z$ events. The figure on the left shows the distribution for prompt $J / \psi$ 's and the figure on the right shows nonprompt $J / \psi$ 's. 


\section{Conclusion}

Measurements of quarkonia plus X can be used to calculate the effective cross section $\left(\sigma_{\text {eff }}\right)$; this can give insight into DPS production. The large datasets at the Tevatron and LHC are allowing more measurements to be made in this exciting area.

\section{References}

[1] V. Khachatryan et al. (CMS Collaboration), Measurement of J/Psi and Psi(2S) Prompt DoubleDifferential Cross Sections in pp Collisions at sqrt(s) $=7$ TeV, Phys. Rev. Lett. 114, 191802 (2015).

[2] ATLAS Collaboration, Measurement of the differential cross-sections of prompt and non-prompt production of $\mathrm{J} / \mathrm{Psi}$ and Psi(2S) in pp collisions at sqrt(s) $=7$ and $8 \mathrm{TeV}$ with the ATLAS detector, Eur. Phys. J. C76, 283 (2016).

[3] ATLAS Collaboration,Measurement of the differential non-prompt J/Psi production fraction in sqrt(s) $=13 \mathrm{TeV}$ pp collisions at the ATLAS experiment, ATLAS-CONF-2015-030.

[4] V. Khachatryan et al. (CMS Collaboration), Measurement of prompt J/Psi pair production in pp collisions at sqrt(s) = $7 \mathrm{TeV}$, JHEP 09 (2014) 094.

[5] V.M. Abazov et al. (D0 Collaboration), Observation and studies of double J/Psi production at the Tevatron, PRD 90 111101(R) (2014).

[6] LHCb Collaboration, Observation of J/Psi pair production in pp collisions at sqrt(s) $=7 \mathrm{TeV}, \mathrm{PLB}$ 707 (2012) 52.

[7] V.M. Abazov et al. (D0 Collaboration), Evidence for Simultaneous Production of J/Psi and Upsilon Mesons, PRL 116, 082002 (2016).

[8] CMS Collaboration, Observation of Upsilon (1S) pair production in proton-proton collisions at $\operatorname{sqrt}(s)=8 \mathrm{TeV}$, arXiv:1610.07095, CMS-BPH-14-008, CERN-EP-2016-237.

[9] LHCb Collaboration, Production of associated Upsilon and open charm hadrons in pp collisions at sqrt(s) = 7 and $8 \mathrm{TeV}$ via double parton scattering, JHEP 07 (2016) 052.

[10] ATLAS Collaboration, Observation and measurements of the production of prompt and non-prompt $\mathrm{J} /$ Psi mesons in association with a $\mathrm{Z}$ boson in pp collisions at sqrt(s) $=8 \mathrm{TeV}$ with the ATLAS detector, Eur. Phys. J. C (2015) 75:229; ATLAS Collaboration, Measurement of the production of prompt J/Psi mesons in association with a $\mathrm{W}+/$ - boson in pp collisions at sqrt(s) $=7 \mathrm{TeV}$ with the ATLAS detector, JHEP 04 (2014) 172. 